J. Korean Math. Soc. 49 (2012), No. 3, pp. 449-473

http://dx.doi.org/10.4134/JKMS.2012.49.3.449

\title{
CONTINUOUS ORDER REPRESENTABILITY PROPERTIES OF TOPOLOGICAL SPACES AND ALGEBRAIC STRUCTURES
}

\author{
María Jesús Campión, Juan Carlos Candeal, Esteban Induráin, \\ and Ghanshyam Bhagvandas Mehta
}

\begin{abstract}
In the present paper, we study the relationship between continuous order-representability and the fulfillment of the usual covering properties on topological spaces. We also consider the case of some algebraic structures providing an application of our results to the social choice theory context.
\end{abstract}

\section{Introduction}

A topology $\tau$ defined on a nonempty set $X$ is said to satisfy the continuous representability property (CRP) if every continuous total preorder $\precsim$ defined on $X$ admits a numerical representation by means of a continuous real-valued order-monomorphism (i.e., a continuous map $u: X \rightarrow \mathbb{R}$ such that $x \precsim y \Longleftrightarrow$ $u(x) \leq u(y)$ for every $x, y \in X)$. There are different motivations to study this order-representability property (CRP). It is interesting from a topological point of view because it can be used to characterize other topological properties of the given space $X$. To cite two recent examples, we can say that it has already been used to analyze order-extension properties of topological spaces (see Yi [37] or Campión et al. $[5,7])$, as well as to characterize various classical topological properties of a Banach space (see Campión et al. [4]) in functional analysis.

The study of covering properties on topological totally ordered spaces is classical in the mathematical theory of ordered structures (see e.g. Lutzer and Bennet [29]). A further step consists in considering topological spaces (not necessarily endowed a priori with any ordering) and then investigate the family of all the continuous total preorders that may be defined there. This is equivalent to start from a topology $\tau$ on a nonempty set $X$ and consider the family of all its subtopologies that are preorderable. Obviously, properties of these

Received April 5, 2010; Revised October 27, 2011.

2010 Mathematics Subject Classification. 54F05.

Key words and phrases. ordered structures on topological spaces, order-representability properties, covering properties, preorderable subtopologies, algebraic order-representability, social choice theory. 
preorderable subtopologies will reflect properties of the given topological space $(X, \tau)$, and vice versa. In the present paper we work in this direction, paying special attention to covering properties of the preorderable subtopologies of a given topology, in order to analyze the structure, closely related to those covering properties, of topological spaces that satisfy the continuous orderrepresentability property $(\mathrm{CRP})$.

The structure of the paper goes as follows: after the introduction (Section 1) and preliminaries (Section 2), we include a preparatory Section 3 containing results on the topological properties of order topologies, that will be used in the main Section 4 to achieve a characterization of topological spaces satisfying CRP. The key characterization given in Theorem 4.1 is stated in terms of the fulfillment of the second countability axiom for every preorderable subtopology of the given topology. This implies that any topological property that makes every preorderable subtopology to be second countable provides a sufficient condition for CRP to be held. Properties of this kind appear throughout Section 4. In Section 5, an extension of CRP in the algebraic setting, called continuous algebraic representability property (shortly, CARP), is introduced. Basically, whereas CRP asks for continuous numerical representations, CARP asks for those which are continuous and, in addition, preserve the algebraic structure. In Section 6, we develop an application of the previous algebraic approach to social choice theory.

\section{Preliminaries}

A preorder $\precsim$ on an arbitrary nonempty set $X$ is a binary relation on $X$ which is reflexive and transitive.

An antisymmetric preorder is said to be an order. A total preorder $\precsim$ on a set $X$ is a preorder such that if $x, y \in X$, then $[x \precsim y]$ or $[y \precsim x]$.

If $\precsim$ is a preorder on $X$, then as usual we denote the associated asymmetric relation by $\prec$ and the associated equivalence relation by $\sim$ and these are defined, respectively, by $[x \prec y \Longleftrightarrow(x \precsim y) \wedge \neg(y \precsim x)]$ and $[x \sim y \Longleftrightarrow(x \precsim y) \wedge(y \precsim$ $x)]$. Also, the associated dual preorder $\precsim_{d}$ is defined by $\left[x \precsim_{d} y \Longleftrightarrow y \precsim x\right]$.

Let $(X, \precsim)$ be a totally preordered set and let $X / \sim$ be the set of equivalence classes. If $x \in X$ we denote the equivalence class of $x$ by $[x]$. The preorder $\precsim$ on $X$ induces a natural order $\preceq$ on $X / \sim$ defined by $[x] \preceq[y] \Longleftrightarrow x \precsim y$.

Let $[x],[y]$ be two equivalence classes in $X / \sim$. Then we say that the ordered pair $([x],[y]) \in(X / \sim) \times(X / \sim)$ is a jump if there is no $[z] \in X / \sim$ such that $[x]<[z]<[y]$, where $<$ denotes the asymmetric part of $\preceq$. If $([x],[y])$ is a jump, then we sometimes abuse notation and say that $(x, y)$ is a jump in $X$. But it should be remembered that a jump is only defined for the corresponding equivalence classes.

A totally preordered set $(X, \precsim)$ is said to be densely ordered if it has no jumps. A subset $Z$ of $X$ is said to be order-dense in $X$ with respect to $\precsim$, if 
$x, y \in X$ and $x \prec y$ imply that there exists $z \in Z$ such that $x \precsim z \precsim y$. $(X, \precsim)$ is said to be order-separable if it has a countable order-dense subset.

A totally preordered set $(X, \precsim)$ is said to be Dedekind-complete if each nonempty subset $F$ that has an upper bound has a least upper bound.

Let $(X, \precsim)$ be a totally preordered set. A subset $Z \subseteq X$ is said to be coinitial (respectively, cofinal) in $X$ if for every $x \in X$ there exists some $z \in Z$ such that $z \precsim x$ (respectively, such that $x \precsim z$ ). The preorder $\precsim$ is said to be countably bounded if there exists a countable subset $Z \subseteq X$ that is coinitial and cofinal in $X$.

If $(X, \precsim)$ is a preordered set, then a real-valued function $u: X \rightarrow \mathbb{R}$ is said to be

(i) increasing if for every $x, y \in X,[x \precsim y \Rightarrow u(x) \leq u(y)]$,

(ii) order-preserving if $f$ is increasing and $[x \prec y \Rightarrow u(x)<u(y)]$.

An order-preserving function is also said to be an order-monomorphism.

If a nonempty set $X$ is endowed with a topology $\tau$, then a total preorder $\precsim$ on $X$ is said to be continuously representable if there exists an ordermonomorphism $u: X \rightarrow \mathbb{R}$ that is continuous.

Let $(X, \precsim)$ be a totally preordered set. The family of all sets of the form $L(x)=\{a \in X: a \prec x\}$ and $G(x)=\{a \in X: x \prec a\}$, where $x \in X$ is a subbasis for a topology $\tau_{\precsim}$ on $X$. The pair $\left(X, \tau_{\precsim}\right)$ is called the order topology on $X$. The pair $\left(X, \tau_{\precsim}\right)$ is called a preordered topological space.

If $(X, \precsim)$ is a preordered set and $\tau$ is a topology on $X$, then the preorder $\precsim$ is said to be $\tau$-continuous on $X$ if for each $x \in X$ the sets $\{a \in X: x \precsim a\}$ and $\{b \in X: b \precsim x\}$ are $\tau$-closed in $X$. If $\precsim$ is a total preorder on $X$, then it is easy to prove that the continuity of $\precsim$ amounts to the fact that the graph of $\precsim$ (i.e., $\{(x, y) \in X \times X ; x \precsim y\})$ is a closed subset of $X^{2}=X \times X$ endowed with the product topology $\tau_{X} \times \tau_{X}$. A topology $\tau$ on $(X, \precsim)$ is said to be natural if $\precsim$ is $\tau$-continuous. Thus, if $\precsim$ is continuous with respect to a topology $\tau$ on $X$, then $\tau_{\precsim} \subseteq \tau$.

Given a nonempty set $X$ endowed with a topology $\tau$ (i.e., $(X, \tau)$ is a topological space), the topology $\tau$ on $X$ is said to have the continuous representability property $(\mathrm{CRP})$ if every continuous total preorder $\precsim$ defined on $X$ admits a representation by means of a continuous order-monomorphism. Topologies of this kind were introduced by Herden [25] under the name of "useful topologies" (see also Herden and Pallack [26]). Among the topologies that have CRP are the second countable ones (see Debreu [17]), the connected plus separable ones (see Eilenberg [19]) and the locally connected plus separable ones (see Campión et al. [8]).

Given a topological space $(X, \tau)$, the topology $\tau$ is said to be separably connected if for every two points $a, b \in X$ there exists a connected and separable subset $C_{a, b} \subseteq X$ that includes $a$ and $b$, and it is said to be preorderable if it is the order topology $\tau \precsim$ of some total preorder $\precsim$ defined on $X$. 
Remark 2.1. At this point, it is important to explain why this theory of orderrepresentability of topological spaces deals with total preorders instead of (just) total orders. The main reason is that on many classical topological spaces $(X, \tau)$ there is no continuous total order (A well known example of this situation is the real plane $\mathbb{R}^{2}$ endowed with its usual Euclidean metric and topology, see Theorem 4 in Candeal and Induráin [10] for details).

\section{Properties of order topologies}

Before studying order-representability properties on a (general) topological space, it seems necessary to begin with a totally preordered set $(X, \precsim)$ endowed with its corresponding order topology $\tau_{\precsim}$, in order to understand better what else must happen for the order topology $\tau_{\precsim}$ to have some classical topological property.

Having this idea in mind, we include this preparatory Section 3, in which we quote several helpful results on ordered sets, to be used in the sequel. Some proofs have been omitted because they are either straightforward or well-known (see e.g. Bridges and Mehta [3], Th. 1.6.11 and Th. 3.2.9; Campión et al. [5], Lemma 3.9 and Corollary 3.10; Steen and Seebach [35], pp. 67-68).

Lemma 3.1. Let $X$ be a nonempty set endowed with a total preorder $\precsim$. Let $\tau_{\precsim}$ be the order topology on $X$. Then the following conditions are equivalent:

(i) The total preorder $\precsim$ is continuously representable through an ordermonomorphism.

(ii) The order topology $\tau_{\precsim}$ is second countable.

(iii) The totally preordered set $(X, \precsim)$ is order-separable.

Lemma 3.2. Let $X$ be a nonempty set endowed with a total order $\preceq$.

(i) The order topology $\tau_{\preceq}$ is connected if and only if $(X, \preceq)$ is densely ordered and Dedekind complete.

(ii) The order topology $\tau_{\preceq}$ on $X$ is second countable if and only if it is metrizable and separāble.

(iii) If the order topology $\tau_{\preceq}$ on $X$ is connected and separable, then it is second countable.

Remark 3.3. Separability cannot be dropped in part (ii) of Lemma 3.2. A counterexample is $X=(0,1) \times(0,1) \subset \mathbb{R}^{2}$ endowed with the lexicographic order $\leq_{L}$ defined by $(x, y) \leq_{L}(z, t) \Longleftrightarrow[(x<z) \vee(x=z, y \leq t)]$ for every $0<x, y, z, t<1$ (see also Theorem 2 in Candeal and Induráin [12]).

Lemma 3.4. Let $X$ be a nonempty set endowed with a total order $\preceq$. Then the order topology $\tau_{\preceq}$ on $X$ is path-connected if and only if it is separably connected.

Proof. See part (iv) of Remark 2 in Candeal et al. [9].

Remark 3.5. On a general topological space $(X, \tau)$ it is clear that connected plus separable implies separably connected. The converse may fail to be true 
(consider, e.g., a non-separable Banach space endowed with the norm topology). It is also true that separably connected implies connected, but, even on metric spaces, the converse is not true in general (see, e.g., Simon [34]). There are also examples of separably connected topological spaces that are not path-connected: Consider, for instance, the subset of $\mathbb{R}^{2}$ given by $(\{0\} \times$ $[-1,1]) \bigcup\left\{\left(x, \sin \left(\frac{1}{x}\right)\right): x>0\right\}$.

Lemma 3.6. Let $X$ be a nonempty set endowed with a total order $\preceq$ that is countably bounded. Suppose that the order topology $\tau_{\preceq}$ on $X$ is separably connected. Then $\tau \preceq$ is second countable.

Proof. ${ }^{1}$ Since $X$ is countably bounded, it can be written as the union of a countable number of nested intervals $\left[a_{n}, b_{n}\right] \subseteq X$. Since $\preceq$ is continuous and $\tau_{\preceq}$ is separably connected each of these intervals is contained in a separable and connected subset of $X$. That means that $X$ is the union of a countable number of connected separable subsets, all of which intersect, so it follows that the order topology $\tau \preceq$ on $X$ is separable and connected. Therefore $\tau \preceq$ is second countable by Lemma 3.2(iii).

Corollary 3.7. Let $X$ be a nonempty set endowed with a topology $\tau$ such that $(X, \tau)$ is separably connected. Let $\precsim$ be a countably bounded and $\tau$-continuous total preorder defined on $X$. Then $\precsim$ is continuously representable through an order-monomorphism.

Proof. Since the topological space $(X, \tau)$ is separably connected, the order topology $\tau_{\precsim}$, that satisfies $\tau_{\precsim} \subseteq \tau$ by hypothesis, is also separably connected. It is obvious that the total order $\preceq$ on the quotient space through indifference $X / \sim$ is also countably bounded, and its corresponding order topology is separably connected. Thus, as a direct consequence of Lemma 3.6 and Lemma 3.1, it follows that $\preceq$ is continuously representable (considering the order topology $\tau \preceq$ on $X / \sim$ and the usual topology on $\mathbb{R}$ ) by means of an order-monomorphism. Consequently, $\precsim$ is continuously representable (now considering the topology $\tau \precsim$ on $X$ and the usual topology on $\mathbb{R}$ ) through an order-monomorphism $\phi$. Indeed, since $\tau_{\precsim} \subseteq \tau$, the map $\phi$ is also continuous if we consider on $X$ the given topology $\sim$ and on $\mathbb{R}$ the usual topology.

Theorem 3.8. Let $X$ be a nonempty set endowed with a total order $\preceq$. Suppose that the order topology $\tau_{\preceq}$ on $X$ is separably connected. Then the following conditions are equivalent:

(i) $\preceq$ is countably bounded.

(ii) $\preceq$ is continuously representable.

(iii) $\tau_{\preceq}$ is second countable.

(iv) $\tau_{\preceq}$ is metrizable and second countable.

(v) $\tau_{\preceq}$ is metrizable and separable.

(vi) $\tau_{\preceq}$ is separable.

\footnotetext{
${ }^{1}$ We owe this proof of Lemma 3.6 to an anonymous referee.
} 
(vii) $\tau \preceq$ is Lindelöf.

(viii) $\tau_{\preceq}$ satisfies the countable chain condition $(C C C)$.

(ix) $\tau_{\preceq}$ is $\sigma$-compact.

Proof. We follow the scheme (i) $\Rightarrow$ (iii); (ii) $\Longleftrightarrow$ (iii) $\Longleftrightarrow$ (iv) $\Longleftrightarrow$ (v); (v) $\Rightarrow$ (vi) $\Rightarrow$ (ii); (iii) $\Rightarrow$ (vii) $\Rightarrow$ (i); (iii) $\Rightarrow$ (viii) $\Rightarrow$ (i) $\Rightarrow$ (ix) $\Rightarrow$ (vii).

The fact (i) $\Rightarrow$ (iii) has been proved in Lemma 3.6.

The equivalence (ii) $\Longleftrightarrow$ (iii) was stated in Lemma 3.1. The order topology $\tau_{\preceq}$ is connected because, by hypothesis, it is separably connected.

The equivalences (iii) $\Longleftrightarrow$ (iv) $\Longleftrightarrow$ (v) as well as the implication (v) $\Rightarrow$ (vi) follow now immediately from Lemma 3.2(ii)-(iii) and Th. 5.6 in Dugundji [18].

Assume now that (vi) holds. Let $D \subseteq X$ be a countable subset that meets

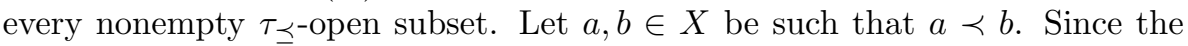
topology $\tau_{\preceq}$ is connected by hypothesis, it follows by Lemma 3.2(i) that $(X, \preceq)$ is densely ordered. Hence there exists $c \in X$ such that $a \prec c \prec b$, so that in particular the $\tau \prec$-open subset $(a, b)=\{x \in X: a \prec x \prec b\}$ is nonempty. Since

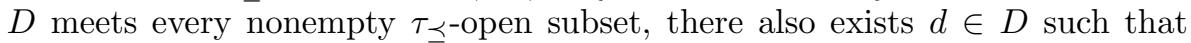
$a \prec d \prec b$. In particular, we have $a \preceq d \preceq b$. Hence $D$ is order-dense in $X$. Condition (ii) follows now from Lemma 3.1.

The fact (iii) $\Rightarrow$ (vii) is immediate.

Assume now that (vii) holds. If $X$ has both a first element $a$ and a last element $b$, then it is plain that $\preceq$ is countably bounded. If $X$ is unbounded, assuming that $X$ has no last element with respect to $\preceq$, it happens that the family $\mathcal{L}$ of all sets of the form $L(x)=\{a \in X: a \prec x\}$ is a $\tau_{\preceq \text {-open covering }}$ of $X$. By hypothesis, there exists a countable subcovering $\left\{L\left(x_{n}\right) n \in \mathbb{N}\right\}$ of $\mathcal{L}$. Similarly, if $X$ has no first element with respect to $\preceq$, then the family $\mathcal{G}$ of all sets of the form $G(y)=\{b \in X: y \prec b\}$ is a $\tau \preceq$-open covering of $X$, and there exists a countable subcovering $\left\{G\left(y_{k}\right) k \in \mathbb{N}\right\}$ of $\mathcal{G}$. Now it is straightforward to see that the countable subset $D=\left\{x_{n} n \in \mathbb{N}\right\} \bigcup\left\{y_{k} k \in \mathbb{N}\right\} \bigcup\{a, b\} \subseteq X$, where $a$ (respectively, $b$ ) denotes the first element if any (respectively, the last element if any) of $X$, is coinitial and cofinal in $X$ with respect to $\preceq$. Therefore, $\preceq$ is countably bounded, so that we get (i).

The fact (iii) $\Rightarrow$ (viii) is clear because every second countable space is separable, and every separable space satisfies the countable chain condition (see e.g. Corollary 2.3.18 in Engelking [20]).

Assume now that (viii) holds. Suppose that there is no countable subset $D \subset X$ that is cofinal in $X$. Then $(X, \preceq)$ contains a subset $Y$ such that $(Y, \preceq)$ can be identified to the first uncountable ordinal $\omega_{1}$ endowed with the ordinal inclusion $\leq$. Thus, there exists a bijective map $F: \omega_{1} \rightarrow Y$ such that $z \preceq t \Longleftrightarrow F^{-1}(z) \leq F^{-1}(t) \quad(z, t \in Y)$. For every $\alpha<\omega_{1}$, let $\alpha+1$ be the ordinal that follows $\alpha$. Since the order topology $\tau \preceq$ on $X$ is separably connected, hence connected, the collection $\left\{(F(\alpha), F(\alpha+\overline{1})): \alpha<\omega_{1}\right\}$ would then violate $\mathrm{CCC}$, which is a contradiction. Therefore, there exist countable 
subsets $C, E \subseteq X$ such that $C$ is coinitial in $X$ and $E$ is cofinal in $X$ with respect to $\preceq$. The union $D=C \cup E$ is countable, coinitial and cofinal in $X$. Thus, $\preceq$ is countably bounded, so that we get (i) again.

Assume now that (i) holds. Let $D=\left\{x_{n}: n \in \mathbb{N}\right\}$ be a coinitial and cofinal subset of $X$. For every $k \neq l \in \mathbb{N}$ such that $x_{k} \prec x_{l}$, we observe that the subset $\left[x_{k}, x_{l}\right]=\left\{y \in X: x_{k} \preceq y \preceq x_{l}\right\}$ is compact in the order topology $\tau_{\preceq}$ of $X$, by Lemma 3.2(i) and Theorem 27.1 in [30]. Therefore $X=$ $\bigcup_{\{k, l \in \mathbb{N}, k \neq l\}}\left[x_{k}, x_{l}\right]$ is obviously $\sigma$-compact. Thus we get (ix). Moreover, since a $\sigma$-compact topological space is in particular Lindelöf we also get (vii).

Remarks 3.9. (i) New conditions could be added to the statement of Theorem 3.8, as a consequence of some results in Sections 2 and 3 of Lutzer and Bennet [29]. These results state that:

Let $X$ be a nonempty set endowed with a total order $\preceq$. Consider on $X$ the order topology $\tau_{\preceq}$. Then it holds that

a) the order topology $\tau \preceq$ satisfies CCC if and only if it is hereditarily Lindelöf,

b) the order topology $\tau_{\preceq}$ is separable if and only if it is hereditarily separable.

Notice in addition that in this result it is not necessary to ask the order topology $\tau_{\prec}$ to be separably connected.

(ii) The hypothesis of being separably connected that appears in the statement of Theorem 3.8 cannot be replaced by the weaker one of connectedness. An example is the lexicographically ordered set $L=\left\{\left[0, \omega_{1}\right) \times J\right\} \cup\left\{\left(\omega_{1}, 0\right)\right\}$ where $J=\{x \in \mathbb{R}: 0 \leq x<1\}$ and $\left\{\left[0, \omega_{1}\right)\right.$ denotes the long line (see, e.g., Steen and Seebach [35]).

\section{The continuous representability property on topological spaces}

In the present section we search for topological conditions on a topological space $(X, \tau)$, in order for $\tau$ to satisfy the continuous representability property (CRP). We pay an special attention to conditions related to covering properties.

The results of Section 3 are decisive in this process, due to the following key fact stated in the next Theorem 4.1, whose proof appears in Campión et al. [6].

Theorem 4.1. Let $(X, \tau)$ be a topological space. Then the topology $\tau$ satisfies CRP if and only if all its preorderable subtopologies are second countable.

A straightforward strengthening of Lemma 3.2(ii) shows that, given a topological space $(X, \tau)$, any preorderable subtopology is second countable if and only if it is separable and pseudometrizable. This fact immediately leads to the following corollary.

Corollary 4.2. Let $(X, \tau)$ be a topological space. Then the topology $\tau$ satisfies CRP if and only if all its preorderable subtopologies are separable and pseudometrizable.

Preorderable topologies were characterized in Campión et al. [6] completing the panorama on orderability of topologies (see Van Dalen and Wattel [36], Purisch [31] or Gutev [24]). 
Suppose now that we are looking for conditions on a topological space $(X, \tau)$ that imply that the topology $\tau$ satisfies CRP. Which kind of conditions should we analyze first?

A glance to Theorem 4.1 gives us an idea to begin with: Any topological condition that implies second countability and is inherited by subtopologies fits well our purposes. We can say even more, observing that any topological property that implies that every preorderable subtopology is second countable also implies CRP.

Now we obtain important results about the property CRP on topological spaces.

Theorem 4.3. Let $(X, \tau)$ be a topological space. Each of the following conditions implies that $(X, \tau)$ satisfies $C R P$ :

(i) $\tau$ is connected and separable (see Eilenberg [19]),

(ii) $\tau$ is separably connected and satisfies CCC (see Campión et al. [4]),

(iii) $\tau$ is separably connected and compact,

(iv) $\tau$ is separably connected and $\sigma$-compact,

(v) $\tau$ is separably connected and satisfies the Lindelöf property,

(vi) $\tau$ is second countable (see Debreu [17]),

(vii) $\tau$ is locally connected and separable (see Candeal et al. [15]).

Proof. Some of these results are already known in the literature. However, we offer here an alternative proof for some of them (parts (i) to (v)) that follows from the previous results of Section 3 and the observation above. Thus, notice that if $\tau$ is connected and separable, then every preorderable subtopology is also connected and separable. Hence, by Lemma 3.2(iii), the preorderable subtopologies of $\tau$ are indeed second countable. In the same way, if $\tau$ is separably connected, then every preorderable subtopology of $\tau$ is also separably connected. If, in addition, $\tau$ satisfies CCC, or the Lindelöf property, or it is compact or $\sigma$-compact, the same happens for every preorderable subtopology of $\tau$. In each of these situations the preorderable subtopologies are actually second countable, as a consequence of Theorem 3.8.

By the way, part (i) is a well-known classical result in the theory of ordered structures (see Eilenberg [19]). The same happens to part (vi), that is known as Debreu's theorem (see Debreu [17] or Bridges and Mehta [3], Ch. 3). Finally, part (ii) also appears in Campión et al. [4], and a proof of part (vii) can be seen in Candeal et al. [14].

Remark 4.4. Part (ii) of the above Theorem 4.3 shows that in separably connected topological spaces CCC implies CRP. However, the converse is not true. An example is the Alexandroff topology on $[0,1] \times[0,1]$ (see Steen and Seebach [35], pp. 120-121 for details) that is compact and path-connected, so that it is separably connected, and, by part (iii) of Theorem 4.3, it satisfies CRP. Moreover, it does not satisfy CCC and fails to be first countable. 
In the category of metric spaces the next Theorem 4.5, and its subsequent Corollary 4.6, provide not only sufficient conditions, but actually characterizations of the fulfillment of the continuous representability property CRP. As a matter of fact, Theorem 4.5 is well-known in this literature (see e.g. Estévez and Hervés [21] or Candeal et al. [9]).

Theorem 4.5. Let $(X, d)$ be a metric space. Then the metric topology $\tau_{d}$ satisfies CRP if and only if it is separable.

Corollary 4.6. (i) Let $(G, \tau)$ be a first countable topological group. Then the topology $\tau$ satisfies CRP if and only if it is separable.

(ii) Let $(G, \tau)$ be a locally compact topological group. Let $C_{0}(G)$ be the space of continuous complex functions defined on $G$ which vanish at infinity. Suppose that the weak topology on $C_{0}(G)$ satisfies the Lindelof property. Then the topology $\tau$ satisfies CRP if and only if it is separable.

(iii) Let $(G, \tau)$ be a locally compact topological group such that the weak topology on $C_{0}(G)$ is normal. Then the topology $\tau$ satisfies CRP if and only if it is separable.

(iv) In a separably connected metric space CCC and CRP are equivalent conditions.

Proof. (i). The classical Birkhoff-Kakutani theorem (see Birkhoff [2], Kakutani [27] or Kelley [28], p. 186) states that on a topological group $(G, \tau)$ the topology $\tau$ is first countable if and only if it is metrizable. The result follows now by Theorem 4.5.

(ii) and (iii). This also follows from Theorem 4.5, making use of Theorem 2 in Corson [16] where it is proved that on a locally compact topological group $(G, \tau)$ the following conditions are equivalent:

(a) $\tau$ is a metrizable topology on $G$,

(b) the weak topology on $C_{0}(G)$ satisfies the Lindelöf property,

(c) the weak topology on $C_{0}(G)$ is normal.

(iv). It is a consequence of Theorem 4.3(ii) and Theorem 4.5, since separability implies CCC.

Remarks 4.7. (i) We cannot drop "first countable" in the statement of Corollary 4.6. In other words, for a topological group $(G, \tau)$ it is not true, in general, that CRP is equivalent to separability. To cite an example, consider an infinite dimensional nonseparable Banach space $X$ (e.g. the Hilbert space of uncountable basis $\left.\ell_{2}(\mathbb{R})\right)$ endowed with the weak topology $\omega$. Observe that $(X, \omega)$ is, in particular, a topological group. The topology $\omega$ is not first countable since otherwise $X$ would be finite dimensional (see e.g. Theorem 6.30 in Aliprantis and Border [1]). On the other hand, $\omega$ is not separable because on a Banach space, and as an easy consequence of Hahn-Banach theorem, the separability of the norm topology is equivalent to the separability of the weak topology $\omega$. Finally, notice that by Theorem 4.3 (ii) $\omega$ satisfies CRP, since it satisfies CCC, as proved in Campión et al. [4]. 
(ii) It could be interesting to say that there are classical topologies on remarkable families of topological spaces that do satisfy CRP. Sometimes this information is easily obtained from the results just introduced in this Section 4. Observe, for instance, that in the category of topological vector spaces any compatible topology is path-connected, hence separably connected. This allows us to use Theorem 4.3 to obtain particular results about the fulfillment of CRP in those spaces.

An important particular case is that of Banach spaces. Thus, if $X$ is a real Banach space, it can be proved that the norm topology $\tau_{\|\cdot\|}$ on $X$ satisfies CRP if and only if it is separable, as a direct consequence of Theorem 4.5. In addition, the weak topology $\omega$ on $X$ always satisfies CRP (see Campión et al. [4]). Hence any topology $\tau$ on $X$ such that $\tau \subseteq \omega$ satisfies CRP, and also the weak star topology $\omega^{*}$ on the Banach space $X^{*}$ (the topological dual of $X$ ) satisfies CRP. Finally, in Candeal et al. [13], pp. 57-59, it has been proved that if the norm topology $\tau_{\|\cdot\|}$ on $X$ is separable, then any topology $\tau$ on the dual Banach space $X^{*}$ such that $\tau \subseteq m\left(X^{*}, X\right)$ satisfies CRP, where $m\left(X^{*}, X\right)$ stands here for the Mackey topology that the dual pair $\left(X^{*}, X\right)$ induces on $X^{*}$.

We conclude this section with a useful result concerning the behaviour of CRP through continuous surjections of topological spaces.

Theorem 4.8. CRP is invariant under continuous surjections.

Proof. Let $\left(X, \tau_{X}\right)$ be a topological space such that $\tau_{X}$ satisfies CRP. Let $\left(Y, \tau_{Y}\right)$ be a topological space such that there exists a continuous surjection $\pi: X \rightarrow Y$. We observe that a total preorder $\precsim_{Y}$ on $Y$ induces a total preorder $\precsim_{X}$ on $X$ by declaring that $x_{1} \precsim_{X} x_{2} \Longleftrightarrow \pi\left(x_{1}\right) \precsim_{Y} \pi\left(x_{2}\right)$. It is clear that, if the preorder $\precsim_{Y}$ is $\tau_{Y}$-continuous, then the corresponding preorder $\precsim_{X}$ on $X$ is $\tau_{X}$-continuous, as a consequence of the continuity of the projection $\pi$ : Given an element $x \in X$, it follows that $\left\{z \in X: z \prec_{X} x\right\}=\left\{z \in X: \pi(z) \prec_{Y}\right.$ $\pi(x)\}=\pi^{-1}\left(\left\{s \in Y: s \prec_{Y} \pi(x)\right\}\right)$. Similarly $\left\{t \in X: x \prec_{X} t\right\}=\{t \in X:$ $\left.\pi(x) \prec_{Y} \pi(t)\right\}=\pi^{-1}\left(\left\{r \in Y: \pi(x) \prec_{Y} r\right\}\right)$. In addition, since $\pi$ is a surjection we have that given an element $y \in Y$ there exists at least one element $x_{y} \in X$ such that $\pi\left(x_{y}\right)=y$. Moreover $\pi\left(\left\{z \in X: z \prec_{X} x_{y}\right\}\right)=\left\{s \in Y: s \prec_{Y} y\right\}$ and $\pi\left(\left\{t \in X: x_{y} \prec_{X} t\right\}\right)=\left\{r \in Y: y \prec_{Y} r\right\}$. Therefore, if we consider on $X$ the order topology induced by $\precsim X$ and on $Y$ the order topology induced by $\precsim_{Y}$, then with respect to these topologies the map $\pi$ is not only a continuous surjection, but also an open map. Since $\tau_{X}$ satisfies CRP, the order topology that $\precsim_{X}$ induces on $X$ is second countable by Theorem 4.1. Hence the order topology that $\precsim_{Y}$ induces on $Y$ is also second countable, because second countability is indeed invariant under continuous open surjections (see e.g. Dugundji [18], Th. 6.2 on p. 174). Again by Theorem 4.1, we conclude that $\tau_{Y}$ satisfies CRP.

Corollary 4.9. (i) CRP is invariant under topological quotients. 
(ii) If the product topology $\tau_{p}$ of a product $\Pi_{i \in I}\left(X_{i}, \tau_{i}\right)$ of topological spaces satisfies CRP, then the topology $\tau_{i}(i \in I)$ of each factor also satisfies CRP.

Remark 4.10. The converse of part (ii) of Corollary 4.9 is an open question.

In this direction, we say that a property $\mathcal{P}$ on topological spaces is said to be multiplicative if for any topological spaces $\left(X, \tau_{X}\right)$ and $\left(Y, \tau_{Y}\right)$ that satisfy $\mathcal{P}$, the topological space $\left(X \times Y, \tau_{X} \times \tau_{Y}\right)$ also satisfies $\mathcal{P}$. Similarly, $\mathcal{P}$ is said to be a square property if for any topological space $\left(X, \tau_{X}\right)$ that satisfies $\mathcal{P}$, the square topological space $\left(X \times X, \tau_{X} \times \tau_{X}\right)$ also satisfies $\mathcal{P}$. Of course, multiplicative properties are in particular square properties but the converse is not true, in general. Now, the open problem is the following:

(i) Is CRP a multiplicative property?

(ii) Is CRP a square property?

Of course, there are partial answers to the aforementioned question. For instance, as a consequence of Theorem 4.5, on metric spaces CRP is a multiplicative property because separability and metrizability are indeed multiplicative properties. Related to the open problem just introduced we may conjecture that CRP is a multiplicative property on separably connected topological spaces.

\section{The continuous algebraic representability property}

In this section, we explore the algebraic version of CRP. In the topological setting, CRP appears whenever we look for ordinal representations of total preorders that, in addition, preserve a nice topological property: namely, the continuity. In the algebraic context, in addition to the continuity property for an order-preserving function, we will ask for a new demanding requirement: that of being an algebraic-homomorphism. Of course, this imposes some kind of compatibility among order, topology, and algebra involved. Although we could begin with a very simple algebraic ordered structure (e.g., think of a totally preordered semigroup), we will focus on richer algebraic systems. In particular, we will pay attention to totally preordered real algebras. The reason for this choice will be clarified later on in Section 6 when all of this algebraic machinery will be applied to characterize strongly dictatorial social welfare functionals, an important issue within social choice theory. We now introduce some basic definitions.

Definition 5.1. A real algebra $(X,+, \cdot \mathbb{R}, *)$ is a set $X$ endowed with three binary operations so that:

(i) $(X,+, \cdot \mathbb{R})$ is a real vector space.

(ii) $(X,+, *)$ is a ring.

(iii) $\lambda \cdot(x * y)=(\lambda \cdot x) * y=x *(\lambda \cdot y)$ for all $x, y \in X, \lambda \in \mathbb{R}$.

Notation. The null element of $X$ with respect to "+" will be denoted by $\mathbf{0}$. 
Let $\left(X,+, \cdot{ }_{\mathbb{R}}, *\right),\left(\bar{X}, \overline{+},{ }^{\cdot} \mathbb{R}, \bar{*}\right)$ be two real algebras. A function $v: X \longrightarrow \bar{X}$ is said to be an algebra-homomorphism if the following three conditions are met:

(a) $v(x+y)=v(x) \mp v(y)$ for all $x, y \in X$ (i.e., it is additive),

(b) $v(\lambda \cdot x)=\lambda \cdot v(x)$ for all $x \in X, \lambda \in \mathbb{R}$ (i.e., it is homogeneous),

(c) $v(x * y)=v(x) \bar{*} v(y)$ for all $x, y \in X$ (i.e., it is multiplicative).

Remark 5.2. Condition (a) in combination to condition (b) can be given in a single formula in the following terms:

$v(\lambda \cdot x+\beta \cdot y)=\lambda \cdot v(x) \mp \beta \tau v(y)$ for all $x, y \in X, \lambda, \beta \in \mathbb{R}$ (i.e., it is linear).

In the next definition, some usual compatibility conditions between order and algebra are introduced. This leads to the concept of a totally preordered real algebra, or a totally ordered real algebra if the preorder is an order.

Definition 5.3. A totally preordered real algebra $(X, \precsim,+, \cdot \mathbb{R}, *)$ is a real algebra equipped with a total preorder $\precsim$ which is compatible with the operations "+", "• $\mathbb{R}$ and "*", i.e., if the following three conditions are met:

(1) $x \precsim y$ implies $x+z \precsim y+z$ for all $z \in X$ (translation-invariance),

(2) $x \precsim y, 0 \leq \lambda$ imply $\lambda \cdot x \precsim \lambda \cdot y$ (homotheticity),

(3) $x \precsim y, \mathbf{0} \precsim z$ imply $z * x \precsim z * y$ and $x * z \precsim y * z$ (multiplicativeinvariance).

Notation. The class of all total preorders defined on a real algebra $X$ that satisfy the conditions (1) to (3) of Definition 5.3 above will be denoted by $\mathcal{P}_{a}$, i.e., $\mathcal{P}_{a}=\{\precsim$ : $\precsim$ is a translation-invariant, homothetic and multiplicativeinvariant total preorder defined on $X\}$.

Remark 5.4. An easy example of a totally preordered real algebra is the $n$ dimensional Euclidean space $X=\mathbb{R}^{n}, n \geq 1$, endowed with the usual binary operations, "+", "* $\mathbb{R}$ " and " $*$ ", defined componentwise (i.e., $\left(a_{i}\right)+\left(b_{i}\right)=\left(a_{i}+\right.$ $\left.b_{i}\right), \lambda \cdot\left(a_{i}\right)=\left(\lambda a_{i}\right),\left(a_{i}\right) *\left(b_{i}\right)=\left(a_{i} b_{i}\right)$ for all $\left.\left(a_{i}\right),\left(b_{i}\right) \in \mathbb{R}^{n}, \lambda \in \mathbb{R}\right)$, and the total preorder defined as follows: Let $j \in\{1, \ldots, n\}$ be fixed. Then, for every $\left(x_{i}\right),\left(y_{i}\right) \in \mathbb{R}^{n}$, define $\left(x_{i}\right) \precsim\left(y_{i}\right)$ if and only if $x_{j} \leq y_{j}$. This total preorder only pays attention to the $j$-th coordinate of the corresponding vectors. This is the reason why we call it a projective total preorder on $\mathbb{R}^{n}$. This example can be straightforwardly generalized to include infinite-dimensional sequence spaces like $X=c_{0}$ or $X=l_{1}$, standard notations which correspond to the Banach space of real sequences vanishing at infinity and the Banach space of absolutely sumable real sequences, respectively.

An obvious example of a non-projective total preorder $\precsim$ defined on $X=\mathbb{R}^{n}$ which satisfies the conditions (1) to (3) of Definition 5.3 is the trivial one (i.e., $\left(x_{i}\right) \sim\left(y_{i}\right)$ for every $\left.\left(x_{i}\right),\left(y_{i}\right) \in \mathbb{R}^{n}\right)$. Notice that this particular binary relation belongs to $\mathcal{P}_{a}$ for any real algebra $X$. We will use the notation $\precsim t$ to refer to this total preorder (i.e., $x \sim_{t} y$ for all $x, y \in X$ ). Now, we present a much 
more sophisticated example of a total preorder defined on a sequence space that belongs to $\mathcal{P}_{a}$ and is non-projective. Consider the space $X=l_{\infty}$, which consists of all bounded real sequences. Endowed with the usual operations defined coordinatewise $l_{\infty}$ is a real algebra. Let us denote by $\beta(\mathbb{N})$ the StoneCech compactification of the set of the natural numbers $\mathbb{N}$ (see, e.g., Dugundji [18]). By the Stone-Čech's theorem every $x \in l_{\infty}$ extends in a unique manner to a continuous real-valued function, say, $\tilde{x}$, defined on $\beta(\mathbb{N})$. Let $p \in \beta(\mathbb{N}) \backslash \mathbb{N}$ be fixed. Then, it can be easily proved that the relation defined as $x \precsim_{p} y$ if and only if $\tilde{x}(p) \leq \tilde{y}(p),\left(x, y \in l_{\infty}\right)$, is a total preorder on $l_{\infty}$ that makes it to be a totally preordered real algebra. Clearly, $\precsim_{p}$, so defined, is not projective.

Next we define the notion of a topological real algebra.

Definition 5.5. A real algebra $(X,+, \cdot \mathbb{R}, *)$ equipped with a topology $\tau$ is said to be a topological real algebra if $(X, \tau,+, \cdot \mathbb{R})$ is a topological vector space and $*$ is a continuous binary operation on $X \times X$.

Remark 5.6. Examples of topological real algebras are $\mathbb{R}^{n}, n \geq 1$, with the usual binary operations mentioned above and the Euclidean topology. Also, they are $c_{0}, l_{\infty}$ and $l_{1}$ endowed with the topology given by the usual norms; i.e., $\left\|\left(x_{n}\right)\right\|_{\infty}=\sup \left\{\left|x_{n}\right|: n \in \mathbb{N}\right\}$ for any $\left(x_{n}\right) \in c_{0}$ or $l_{\infty}$, and $\left\|\left(x_{n}\right)\right\|_{1}=\sum\left|x_{n}\right|$, for any $\left(x_{n}\right) \in l_{1}$. Actually, all of these are examples of richer mathematical structures; namely, they are Banach algebras (see, e.g., Rickart [32] for an excellent account on this topic).

Notation. In order to shorten the notation, we will simply write $X$ to denote a real algebra. If there is no ambiguity, we will keep the same notation throughout this section even though $X$ is a totally preordered real algebra or a totally preordered topological real algebra.

Before introducing the concept of CARP in this algebraic environment a new notion is needed. A total preorder $\precsim$ on $X$ is said to be zero if $x * y \sim \mathbf{0}$ for all $x, y \in X$. Otherwise $\precsim$ is said to be non-zero. If $\precsim$ is a total order, then $\sim$ is the equality relation $=$. In this case, since the previous condition reduces to $x * y=\mathbf{0}$ for all $x, y \in X$, we will say that $X$ is zero or non-zero, respectively.

Definition 5.7. Let $X$ be a real algebra and let $\tau$ be a topology on $X$. Then $\tau$ satisfies the continuous algebraic representability property (shortly, CARP) if every continuous, translation-invariant, homothetic, multiplicativeinvariant and non-zero total preorder $\precsim$ defined on $X$ admits a continuous order-preserving function which is an algebra-homomorphism (shortly, a continuous algebraic order-preserving function).

Remarks 5.8. (i) It should be observed that the only zero total preorder defined on a real algebra $X$ that admits an algebraic order-preserving function is the trivial one $\precsim_{t}$ (i.e., $x \sim_{t} y$ for every $x, y \in X$ ) for which the algebraic orderpreserving function turns out to be the null function. 
(ii) The condition of being non-zero plays an important role in Definition 5.7. Indeed, by Remark 5.8(i) and from the point of view of the existence of an algebraic order-preserving function, zero and non-trivial total preorders on a real algebra $X$ are pathological. It is remarkable that this kind of total preorders can exist even though $X$ is endowed with a nice topology. This is the reason why they are not included in Definition 5.7. Let us see an example of a topological totally preordered real algebra $(X, \tau, \precsim,+, \cdot \mathbb{R}, *)$ such that $\precsim$ is zero, continuous and non-trivial. Consider $X=\mathbb{R}^{2}$, endowed with the usual Euclidean topology and the usual binary operations, "+" and ". $\mathbb{R}$ " defined componentwise. Then, define both the binary operation "*" and the binary relation $\precsim$ as follows: $\left(x_{1}, x_{2}\right) *\left(y_{1}, y_{2}\right)=\left(x_{1} y_{1}, 0\right) ;\left(x_{1}, x_{2}\right) \precsim\left(y_{1}, y_{2}\right)$ if and only if $x_{2} \leq y_{2}$. It is simple to see that $X$, with " $*$ " so defined, is a topological real algebra. In addition, it can be easily shown that $\precsim$, so defined, is a continuous, zero and non-trivial total preorder on $X$ which belongs to $\mathcal{P}_{a}$. Notice that, although it admits a linear order-preserving function (e.g., $\psi\left(x_{1}, x_{2}\right)=x_{2}$ for every $\left(x_{1}, x_{2}\right) \in \mathbb{R}^{2}$, is such a function), there is no such a function which is multiplicative.

(iii) Projective preorders on $\mathbb{R}^{n}$, or on some sequence spaces like $c_{0}$ or $l_{1}$, have the interesting property that they are the only non-trivial total preorders that admit a continuous algebraic order-preserving function (see, Remark 5.15 below). However, this is not the case for any arbitrary totally preordered topological real algebra. Indeed, consider again the Banach algebra $X=l_{\infty}$ and let $p \in \beta(\mathbb{N}) \backslash \mathbb{N}$ be fixed (see the second paragraph of Remark 5.4 above). Then, $\precsim_{p}$ is a non-trivial total preorder on $l_{\infty}$ that is not projective. Observe that the evaluation map at $p, x \in l_{\infty} \rightsquigarrow e_{p}(x)=\tilde{x}(p) \in \mathbb{R}$, is a continuous algebraic order-preserving function for $\precsim_{p}$.

Notation. Similarly to the notation used above, we now introduce two important sub-classes of binary relations defined on $X$. These are, respectively, $\mathcal{P}=$ $\{\precsim: \precsim$ is a continuous, translation-invariant, homothetic and multiplicativeinvariant total preorder defined on $X\}$ and $\tilde{\mathcal{P}}=\{\precsim: \precsim$ is a non-zero, continuous, translation-invariant, homothetic and multiplicative-invariant total preorder defined on $X\}$. Obviously, $\tilde{\mathcal{P}} \subseteq \mathcal{P}$.

Next lemma will be useful to prove the main result of this section.

Lemma 5.9. Let $X$ be a non-zero ${ }^{2}$ topological real algebra and let $\preceq \in \mathcal{P} a$ total order defined on $X$. Then there is a continuous algebraic order-preserving function for $\preceq$. Moreover, this function is unique, onto and open.

Proof. First of all, we are going to show that the order topology $\tau \preceq$ coincides with $\tau$ on $X$. Notice that, with respect to $\tau, X$ is a Hausdorff space since, by continuity of $\preceq, \tau \preceq \subseteq \tau$ and clearly $X$ is a Hausdorff space with respect

\footnotetext{
${ }^{2}$ We are indebted to an anonymous referee for drawing our attention to include this possibility.
} 
to $\tau \preceq$. Thus, $(X, \tau,+, \cdot \mathbb{R})$ is a Hausdorff topological vector space. Since $\preceq$ is a continuous, translation-invariant and homothetic total (pre-)order on $X$, by a result of Candeal and Induráin [11], there is a linear and continuous orderpreserving function, say $\psi$ for $\preceq$. Notice also that, since $\preceq$ is a total order on $X$, in addition to be both an algebraic and an order isomorphism, $\psi$ is a homeomorphism from $X$ onto the reals. As a direct consequence $(X,+, \cdot \mathbb{R})$ can be identified with $(\mathbb{R},+, \cdot \mathbb{R}), \tau$ with the usual Euclidean topology on $\mathbb{R}$ and $\preceq$ with the usual order $\leq$ on $\mathbb{R}$ or its dual $\leq_{d}$ (i.e., $a \leq_{d} b \Leftrightarrow b \leq a$ for every $a, b \in \mathbb{R})$. In particular, the order topology on $X, \tau_{\preceq}$, coincides with $\tau$.

Now, observe that $(X, \preceq,+, *)$ is a non-zero totally ordered ring $^{3}$ too. In addition, by the argument of the previous paragraph, $\preceq$ is Archimedean (i.e., for any $x, y \in X$, such that $\mathbf{0} \prec x \prec y$, there is $n \in \mathbb{N}$ such that $y \prec n x)$. So, by a result of Pickert and Hion (see Fuchs [23], p. 126), there is an order-preserving function, say $\phi$, for $\preceq$ which is additive (i.e., $\phi(x+y)=\phi(x)+\phi(y)$ for every $x, y \in X)$ and multiplicative. Let us see that $\phi$ meets all of the properties given in the statement of Lemma 5.9. In particular, it remains to prove that, in addition to satisfy the functional equation $\phi(\lambda \cdot x)=\lambda \phi(x)(\lambda \in \mathbb{R}, x \in X)$, $\phi$ is continuous, onto, open and unique.

(1) $\phi$ is continuous: Indeed, by the argument of the first paragraph above, $(X, \preceq)$ can be identified with $(\mathbb{R}, \leq)$ or $\left(\mathbb{R}, \leq_{d}\right)$ and $\tau=\tau_{\preceq}$ with the Euclidean topology on $\mathbb{R}$. Suppose that the first occurs, the other situation being entirely similar. Then $\phi$ turns out to be an increasing function from $\mathbb{R}$ into $\mathbb{R}$ (with the usual meaning of an increasing real-valued function of one real variable). Now, it is well-known that an increasing function from $\mathbb{R}$ into $\mathbb{R}$ is almost everywhere continuous and, in addition, if it is additive, then it is continuous at every point. Therefore, $\phi$ is continuous.

(2) $\phi$ satisfies the functional equation $\phi(\lambda \cdot x)=\lambda \phi(x)(\lambda \in \mathbb{R}, x \in X)$ : Indeed, a standard reasoning proves that, since $\phi$ is additive and continuous, it satisfies this homogeneity equation.

(3) $\phi$ is onto: It follows from linearity.

(4) $\phi$ is open: To show openess let us observe that, since $\phi$ is order-preserving, the image of every open $\preceq$-interval of $X$ is an open $\leq$-interval of $\mathbb{R}$. Now, since $\tau=\tau_{\preceq}$ and $\tau_{\leq}$is the Euclidean topology on $\mathbb{R}$, it directly follows that $\phi$ is open.

(5) $\phi$ is unique: To prove this, we use the fact that $\phi$ is multiplicative. First, it should be noted that $X$ has an identity, say $1_{X}$ (i.e., there is an element, say $1_{X} \in X$, such that $1_{X} * x=x * 1_{X}=x$ for every $\left.x \in X\right)$. Indeed, $1_{X}=\phi^{-1}(1)$ is the identity. In addition, since $\left(X,+, \cdot_{\mathbb{R}}\right)$ is a one-dimensional vector space, for every $x \in X$ there is $\lambda_{x} \in \mathbb{R} ; x=\lambda_{x} \cdot 1_{X}$. Suppose that there is another function $\tilde{\phi}$ that meets the conditions of Lemma 5.9. Let us see that $\tilde{\phi} \equiv \phi$. Indeed, note that, since $\tilde{\phi}$ is multiplicative too, $\tilde{\phi}\left(1_{X}\right)=\phi\left(1_{X}\right)=1$. Then,

\footnotetext{
${ }^{3} \mathrm{~A}$ totally (pre-)ordered ring is a ring endowed with a (left and right) translation-invariant and multiplicative-invariant total (pre-)order.
} 
by linearity, we have that $\tilde{\phi}(x)=\tilde{\phi}\left(\lambda_{x} \cdot 1_{X}\right)=\lambda_{x} \tilde{\phi}\left(1_{X}\right)=\lambda_{x}=\lambda_{x} \phi\left(1_{X}\right)=$ $\phi\left(\lambda_{x} \cdot 1_{X}\right)=\phi(x)$ for every $x \in X$. So, $\tilde{\phi} \equiv \phi$.

Remark 5.10. Consider $\mathbb{R}$ with the usual binary operations, the usual order $\leq$ and the Euclidean topology. As direct consequences of Lemma 5.9 the following results are obtained:

(i) The only (up to a unique order-preserving, homeomorphism and algebraic isomorphism) non-zero totally ordered topological real algebra is $\mathbb{R}$. In particular, a non-zero totally ordered topological real algebra is a commutative field.

(ii) Let $(X, \preceq,+, \cdot \mathbb{R}, *)$ be a non-zero totally ordered real algebra. Then, $\tau \preceq$ is the only topology on $X$ that makes it to be a non-zero totally ordered topological real algebra.

The main result of this section will be a characterization of the continuous algebraic representability property (CARP); but, this result must be prefaced by a remark.

Remark 5.11. The following well-known facts will be used in the sequel. They take part of the folklore of the theory of topological vector spaces and topological algebras (see, e.g., Schaefer [33] and Rickart [32]).

Let $X$ be a real vector space and let $S \subseteq X$ be a linear subspace of $X$. Consider the equivalence relation $R_{S}$ on $X$ defined as follows: $x R_{S} y$ if and only if $x-y \in S$. Then, the binary operations "+" and " $\mathbb{R}$ " on $X$ are stable under $R_{S}$ and so the quotient space $X / S$ is a real vector space too (the corresponding binary operations on this quotient space are denoted also by "+" and " $\mathbb{R} ")$. If, in addition, $X$ is a topological vector space, with topology $\tau$, then the quotient space $X / S$ is also a topological vector space endowed with the quotient topology, denoted by $\tau_{q}$. Notice that $X / S$ is Hausdorff if and only if $S$ is closed. Consider the projection map, denoted by $p$, defined as follows: $x \in X \rightsquigarrow p(x)=$ $[x] \in X / S$. It is well-known that $p$ is linear, continuous and open (with respect to the topology $\tau$ in $X$ and $\tau_{q}$ in $\left.X / S\right)$.

Moreover, if $X$ is a real algebra and $S \subseteq X$ is, in addition to being a linear subspace, an ideal ${ }^{4}$ of $X$, then the operation "*" is also stable under $R_{S}$ which means that the quotient space $X / S$ is a real algebra (the binary operation of the quotient will also be denoted by "*"). If $X$ is a topological real algebra, with topology $\tau$, and $S \subseteq X$ is a linear subspace and an ideal of $X$, then $X / S$ is also a topological real algebra with respect to the quotient topology $\tau_{q}$. Notice that, in this algebraic context, the projection map $p$ is also multiplicative.

Theorem 5.12. Let $X$ be a real algebra and let $\tau$ be a topology on $X$. Then the following assertions are equivalent:

(i) $\tau$ satisfies $C A R P$.

\footnotetext{
${ }^{4}$ An additive subgroup $I$ of a ring $(A,+, *)$ is said to be an ideal if $x * a \in I, a * x \in I$ for every $x \in I, a \in X$.
} 
(ii) Endowed with the order topology $\tau \precsim, X$ is a topological real algebra for every $\precsim \in \tilde{\mathcal{P}}$.

Proof. For the proof of this result, given a total preorder $\precsim$ on $X$, it will be useful to consider the following set $I(\mathbf{0})=\{\mathrm{x} \in \mathrm{X}: \mathrm{x} \sim \mathbf{0}\}$.

(i) $\Longrightarrow\left(\right.$ ii). Let $\precsim \in \tilde{\mathcal{P}}$. Let us see that, endowed with the order topology $\tau_{\precsim}$, $X$ is a topological real algebra. Since, by hypothesis, $\tau$ satisfies CARP there is a continuous algebraic order-preserving function, say $\phi: X \longrightarrow \mathbb{R}$ for $\precsim$. Notice that $\phi \neq 0$ since $\precsim$ is non-zero. Then, it is routine to check that $I(\mathbf{0})=\phi^{-1}(0)$ is a real vector subspace of $X$ which, in addition, is an ideal of $X$. Consider now the quotient space $X / I(\mathbf{0})$. It is simple to see that, actually, $X / I(\mathbf{0})$ is a totally ordered real algebra with the usual binary operations induced by "+", "• $\mathbb{R}$ " and “*” in the quotient $X / I(\mathbf{0})$, and the total order $\preceq$ defined as $[x] \preceq[y] \Leftrightarrow x \precsim y$. It should be noted that $X / I(\mathbf{0})$ is non-zero since $\precsim$ is non-zero (hence there are $x, y \in X$ such that $x * y \nsim \mathbf{0}$ and, therefore, $[x] *[y]=[x * y] \neq[\mathbf{0}])$. Notice that, by Remark 5.11 above, equipped with the quotient topology $\tau_{q}, X / I(\mathbf{0})$ is a Hausdorff topological real algebra. Clearly, $\preceq$ is a continuous total order on $X / I(\mathbf{0})$. Thus, by Remark 5.10 (i) and (ii), $X / I(\mathbf{0})$ can be identified with the reals and $\tau_{\preceq} \equiv \tau_{q}$. Therefore, endowed with the order topology $\tau_{\preceq}, X / I(\mathbf{0})$ is a non-zero topological real algebra. Let us show that $X$ is a topological real algebra too with respect to $\tau \precsim$.

To that end, consider the projection map $p: X \longrightarrow X / I(\mathbf{0})$. By Remark $5.11 p$ is linear, multiplicative, continuous and open. Also, it is very simple to see that $p$ is order-preserving (i.e., $x \precsim y \Leftrightarrow[x] \preceq[y]$ for every $x, y \in X$ ). Furthermore, $p$ is also continuous and open whenever $X$ is endowed with the order topology $\tau \prec$ and $X / I(\mathbf{0})$ is equipped with the order topology $\tau_{\prec}$.

Let us show that, whenever $X$ is equipped with the order topology, the binary operation $+: X \times X \longrightarrow X$ is a continuous map. The proofs for the remaining operations, namely " $\mathbb{R}^{\prime}$ " and "*", are similar. Let then $C \subseteq X$ be a $\tau \prec$-open subset of $X$. We have to find $\tau \prec$-open subsets, say $A, B \subseteq X$, such that $A+B=\{a+b: a \in A, b \in B\} \subseteq \widetilde{C}$. Since, as mentioned above, $p: X \longrightarrow X / I(\mathbf{0})$ is a $\tau \prec$ to $\tau \prec$ continuous and open map, it follows that $p(C)$

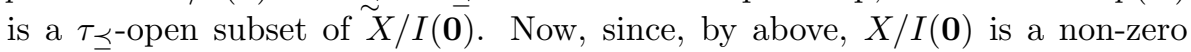

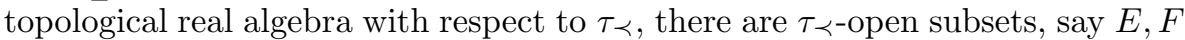
of $X / I(\mathbf{0})$ such that $E+F \subseteq p(C)$. Consider $A=p^{-1}(E)$ and $B=p^{-1}(F)$ and let us prove that $A, B \subseteq X$ are the required subsets. Indeed, $A, B$ are $\tau \precsim$-open subsets of $X$ since $p$ is continuous and $E, F$ are $\tau_{\preceq}$ of $X / I(\mathbf{0})$. It remains to see that $A+B \subseteq C$. To that end, let $a \in A, b \in B$ arbitrarily chosen. Then, there are $e \in E, f \in F$ such that $p(a)=e$ and $p(b)=f$. By linearity, $p(a+b)=p(a)+p(b)=e+f \in p(C)$. This is, there is $c \in C$ such that $p(a+b)=p(c)$ and therefore $a+b \sim c$. This straightforwardly implies that $a+b \in C$ since $C$ is a $\tau \precsim$-open subset of $X$ which, in particular means that if $c \in C$, then $c^{\prime} \in C$ for every $c^{\prime} \sim c$. 
For the converse, (ii) $\Longrightarrow$ (i), we argue as follows. Let $\precsim \in \tilde{\mathcal{P}}$. Let us prove that there is a continuous algebraic order-preserving function for $\precsim$. Consider the order topology $\tau_{\precsim}$ on $X$, so that $\left(X, \tau_{\precsim},+, \cdot \mathbb{R}, *\right)$ is a topological real algebra, by hypothesis.

Let us see first that $I(\mathbf{0})$ is both a linear subspace and an ideal of $X$, for which we need to prove the following two properties:

(a) $I(\mathbf{0})$ is a real vector subspace of $X$.

(b) For every $x \in I(\mathbf{0}), y \in X$, it holds that $x * y \in I(\mathbf{0})$ and $y * x \in I(\mathbf{0})$.

Let $x, y \in I(\mathbf{0})$. Since $\precsim$ is translation-invariant, it follows that $x+y \sim$ $x+\mathbf{0}=\mathrm{x} \sim \mathbf{0}$. So, in order to prove (a), it is sufficient to see that, given $x \in I(\mathbf{0})$ and $\lambda \in \mathbb{R}$, then $\lambda \cdot x \sim \mathbf{0}$. If $\lambda \geq 0$, then, by homotheticity, $\lambda \cdot x \sim \mathbf{0}$. If $\lambda<0$, then $(-\lambda) \cdot x \sim \mathbf{0}$. But $(-\lambda) \cdot x=-\lambda \cdot x$ and, by translation invariance of $\precsim, \lambda \cdot x \sim \mathbf{0}$.

To prove (b), let $y \in X$ and $x \in I(\mathbf{0})$. If $\mathbf{0} \precsim \mathrm{y}$, then, because $\precsim$ is multiplicative-invariant, $x * y \precsim \mathbf{0} * \mathrm{y}=\mathbf{0}$ and $\mathbf{0}=\mathbf{0} * \mathrm{y} \precsim \mathrm{x} * \mathrm{y}$. Therefore, $x * y \sim \mathbf{0}$. If $y \precsim \mathbf{0}$, then $\mathbf{0} \precsim-y$ and so $-x * y \sim \mathbf{0}$. Since $x * y=-(-x) * y$, and $I(\mathbf{0})$ is a vector subspace of $X$, it holds that $x * y \sim \mathbf{0}$. The case $y * x \in I(\mathbf{0})$ is proved in a similar way. Thus (b) holds and therefore $I(\mathbf{0})$ is an ideal of $X$.

Consider again the quotient space $X / I(\mathbf{0})$. It is now routine to see that $X / I(\mathbf{0})$ is a totally ordered real algebra with the usual binary operations induced by " + ", "* $\mathbb{R}$ " and "*" in the quotient $X / I(\mathbf{0})$, and the total order $\preceq$ defined as $[x] \preceq[y] \Leftrightarrow x \precsim y$. As above, $X / I(\mathbf{0})$ is non-zero since, by hypothesis,

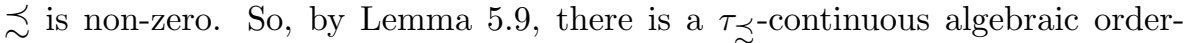
preserving function $u: X / I(\mathbf{0}) \longrightarrow \mathbb{R}$ for $\preceq$. Consider again the projection map $p: X \longrightarrow X / I(\mathbf{0})$. Recall that $p$ is linear, multiplicative, continuous and order-preserving. Then, by considering the composition $\psi=u \circ p: X \longrightarrow \mathbb{R}$ we obtain a $\tau \precsim$-continuous (hence $\tau$-continuous since $\precsim$ is continuous), linear and multiplicative order-preserving function for $\precsim$. Thus $\tau$ satisfies CARP.

Remark 5.13. If $X$ has an identity, say $1 \in X$, then the only zero total preorder on $X$ is the trivial one $\precsim_{t}$. So, if $X$ has an identity, then $\mathcal{P}=\tilde{\mathcal{P}} \bigcup\left\{\precsim_{t}\right\}$. Note that the null function is a continuous algebraic order-preserving function for $\precsim t$. Moreover, for this preorder $\precsim t$, the quotient space $X / I(\mathbf{0})$ is the zero vector (i.e., $X / I(\mathbf{0})=\{[\mathbf{0}]\})$ and therefore the quotient topology amounts to the order topology (which coincides with the discrete topology, $\tau_{d}=\{\emptyset, X\}$, on $X / I(\mathbf{0})$ ). Then, Theorem 5.12 can be re-formulated in the following terms.

Let $X$ be a real algebra with an identity and let $\tau$ be a topology on $X$. Then the following assertions are equivalent:

(i) Every $\precsim \in \mathcal{P}$ admits a continuous algebraic order-preserving function.

(ii) Endowed with the order topology $\tau_{\precsim}, X$ is a topological real algebra for every $\precsim \in \mathcal{P}$.

If $\tau$ is a topology on $X$ that makes it to be a topological real algebra, then we have the following interesting consequence. 
Corollary 5.14. Let $X$ be a real algebra equipped with a topology $\tau$. If $X$ is a topological real algebra, then $\tau$ satisfies CARP.

Proof. According to the statement of Theorem 5.12, we only have to prove that, equipped with the order topology $\tau_{\precsim}, X$ is a topological real algebra for every $\precsim \in \tilde{\mathcal{P}}$. Let then be $\precsim$ such a total preorder on $X$ and consider again the quotient space $X / I(\mathbf{0})$. As seen in the proof of Theorem 5.12, $X / I(\mathbf{0})$ is a non-zero totally ordered topological real algebra. Then, the result follows directly from Remark 5.10(i) and (ii) above and the argument provided in the last paragraph of the proof of the implication (i) $\Longrightarrow$ (ii) of Theorem 5.12.

Remark 5.15. In particular, Corollary 5.14 applies to the $n$-dimensional Euclidean space $\mathbb{R}^{n}$ endowed with the usual binary operations, "+", " $\mathbb{R}$ " and "*", defined componentwise, and the Euclidean topology. Moreover, in this case it can be easily seen that the non-null algebra-homomorphisms are of the form $\psi\left(x_{1}, \ldots, x_{j}, \ldots, x_{n}\right)=x_{j}$ for some $j \in\{1, \ldots, n\}$. Actually, in this case, continuity and order-preserving are redundant properties. Indeed, let $\psi: \mathbb{R}^{n} \longrightarrow \mathbb{R}$ such a function. Then, since $\psi$ is linear, it is of the form $\psi\left(x_{1}, \ldots, x_{i}, \ldots, x_{n}\right)=$ $\sum_{i} a_{i} x_{i}$, where $a_{i} \in \mathbb{R}$ for every $i \in\{1, \ldots, n\}$. Now, let us denote by $\left(e^{i}\right)_{i=1}^{n}$ the canonical basis of $\mathbb{R}^{n}$. Then, since $\psi$ is multiplicative too, in particular we have that $\psi\left(e^{i} * e^{j}\right)=\psi\left(e^{i}\right) \psi\left(e^{j}\right)=a_{i} a_{j}$ for all $i, j$. But, if $i \neq j$, $\psi\left(e^{i} * e^{j}\right)=\psi(\mathbf{0})=0=a_{i} a_{j}$ and $a_{i}^{2}=\psi\left(e^{i}\right) \psi\left(e^{i}\right)=\psi\left(e^{i} * e^{i}\right)=\psi\left(e^{i}\right)=a_{i}$. Therefore, since $\psi$ is non-null, there is $j \in\{1, \ldots, n\}$ such that $a_{j}=1$ and $a_{i}=0$ for $i \neq j$. Thus, $\psi\left(x_{1}, \ldots, x_{j}, \ldots, x_{n}\right)=x_{j}$. By using a similar argument, it is not difficult to prove that this kind of result also holds true for the infinite-dimensional cases $X=c_{0}$ or $X=l_{1}$, but not for $X=l_{\infty}$ (see, Remark 5.4 and Remarks 5.8(iii)).

Note that, by Corollary 5.14, we have proved that any non-zero (or, equivalently in this case, non-trivial) continuous total preorder defined on $\mathbb{R}^{n}$ which is translation-invariant, homothetic and multiplicative-invariant is projective.

\section{Application to social choice theory}

In this section, we develop an application of the algebraic approach presented in the previous Section 5 to the context of utility theory in the social choice framework. A social welfare functional is a map that assigns a preference relation (total preorder) to any profile of individual utilities. In this literature, a utility function refers to any map $u: X \longrightarrow \mathbb{R}$, where $X$ is the choice set. It should be noted that a utility function $u$ generates a total preorder on $X$, denoted by $\precsim_{u}$, defined as follows: $x \precsim_{u} y$ if and only if $u(x) \leq u(y)$ for every $x, y \in X$. We characterize strongly dictatorial social welfare functionals in terms of invariance and continuity properties of such rules, in the spirit of the literature of utility measurability and (inter/intra) personal comparability (see, e.g., Fleurbaey and Hammond [22]). Before presenting the application some definitions and notations are needed. Other insights regarding the potential use 
of algebraic techniques in social choice theory can be seen in Candeal, Induráin and Molina [15].

Let $X$ be a nonempty set (usually called in this context the set of alternatives or the choice set). Let us denote by $\Re$ the class of all total preorders (or preference relations as are usually called in this setting) defined on $X$. Let $n>1$ be a natural number (number of agents or individuals in the society). The set of all functions from $X$ into $\mathbb{R}$ will be denoted by $U$. A utility function for the agent $i$ will be denoted by $u_{i} \in U$. A profile of utility functions, one for each agent, will be denoted by $\left(u_{1}, \ldots, u_{n}\right)$, i.e., it is an element of the usual product of $n$-copies of $U$, denoted by $U^{n}$, which is usually referred to as the set of all possible profiles. The set which consists of the $n$ first natural numbers will be denoted by $N$, i.e., $N=\{1, \ldots, n\}$.

A social welfare functional is a rule $F: U^{n} \rightarrow \Re$ that assigns a preference relation $F\left(u_{1}, \ldots, u_{n}\right) \in \Re$, interpreted as the social preference relation, to any profile $\left(u_{1}, \ldots, u_{n}\right)$ in the domain $U^{n}$. In order to shorten the notation, for a profile $\left(u_{1}, \ldots, u_{n}\right)$ we will use the notation $\left(u_{i}\right)$. Also, $F\left(u_{i}\right)_{s}$ stands for the strict preference (asymmetric relation) associated with $F\left(u_{i}\right)$.

A social welfare functional $F: U^{n} \rightarrow \Re$ is said to be Paretian if, for any pair of alternatives $x, y \in X$ and any profile $\left(u_{i}\right) \in U^{n}$, we have that $u_{i}(x) \leq u_{i}(y)$ for all $i \in N$ implies $x F\left(u_{i}\right) y$ and also that $u_{i}(x)<u_{i}(y)$ for all $i \in N$ implies $x F\left(u_{i}\right)_{s} y$.

A social welfare functional $F: U^{n} \rightarrow \Re$ satisfies the binary independence of irrelevant alternatives condition if, for any pair of alternatives $x, y \in X$ and any pair of profiles $\left(u_{i}\right),\left(v_{i}\right) \in U^{n}$ with the property that $u_{i}(x)=v_{i}(x)$ and $u_{i}(y)=v_{i}(y)$ for all $i \in N$, we have that $x F\left(u_{i}\right) y$ if and only if $x F\left(v_{i}\right) y$.

A social welfare functional $F: U^{n} \longrightarrow \Re$ is called strongly dictatorial if there is an agent $j \in N$ (the dictator) such that $F\left(u_{i}\right)$ coincides with $\precsim u_{j}$ for every $\left(u_{i}\right) \in U^{n}$, where $\precsim u_{j}$ denotes the preference relation on $X$ induced by the utility function of the $j$-agent, $u_{j}$.

A social welfare functional $F: U^{n} \longrightarrow \Re$ is called anonymous if for any pair of profiles $\left(u_{i}\right),\left(v_{i}\right) \in U^{n}$ such that $\left(v_{i}\right)$ is derived from $\left(u_{i}\right)$ by permuting the individuals' utility functions, one has that $F\left(u_{i}\right)$ and $F\left(v_{i}\right)$ coincide.

A social welfare functional $F: U^{n} \longrightarrow \Re$ is called continuous if $\left\{\left(\left(a_{i}\right),\left(b_{i}\right)\right) \in\right.$ $\mathbb{R}^{n} \times \mathbb{R}^{n}$ : there exist $\left(u_{i}\right) \in U^{n}, x, y \in X$ with $u_{i}(x)=a_{i}, u_{i}(y)=b_{i}$ and such that $\left.x F\left(u_{i}\right) y\right\}$ is an Euclidean-closed subset of $\mathbb{R}^{n} \times \mathbb{R}^{n}$.

Finally, a social welfare functional $F: U^{n} \longrightarrow \Re$ satisfies information invariance with respect to cardinal measurability if, for any profile $\left(u_{i}\right) \in U^{n}$, and any $n$-tuple of functions $\left(\phi_{1}, \ldots, \phi_{n}\right)$ such that, for each $i \in N$, the map $\phi_{i}: \mathbb{R} \rightarrow \mathbb{R}$ is of the form $\phi_{i}(t)=a_{i}+b_{i} t \quad(t \in \mathbb{R})$ with $a_{i} \in \mathbb{R}$ and $b_{i}>0$, it holds that $F\left(u_{i}\right)$ and $F\left(\phi_{i} \circ u_{i}\right)$ coincide.

We reach the following result. 
Theorem 6.1. Suppose that $X$ contains at least three elements and let $F$ : $U^{n} \rightarrow \Re$ be a social welfare functional. Then the following assertions are equivalent:

(i) F is Paretian, satisfies the binary independence of irrelevant alternatives condition, continuity, and information invariance with respect to cardinal measurability.

(ii) $F$ is strongly dictatorial.

Proof. Let us prove that (ii) implies (i). Suppose then that $F$ is strongly dictatorial and assume without loss of generality that the $j$-th agent acts as the dictator. It is straightforward to see that $F$ is Paretian and also that it satisfies the binary independence of irrelevant alternatives condition. In order to prove that it satisfies information invariance with respect to cardinal measurability, let $\left(u_{i}\right) \in U^{n}$ a profile of utility functions and let $\left(\phi_{1}, \ldots, \phi_{n}\right)$ be a $n$-tuple of functions so that, for every $i \in N, \phi_{i}(t)=a_{i}+b_{i} t(t \in \mathbb{R})$ with $a_{i} \in \mathbb{R}$ and $b_{i}>0$. Since, by hypothesis, the $j$-th agent is the dictator for $F$, it follows that $F\left(u_{i}\right)=\precsim u_{j}$. Now, consider the profile $\left(\phi_{i} \circ u_{i}\right) \in U^{n}$. Then, $F\left(\phi_{i} \circ u_{i}\right)=\precsim_{\phi_{j} \circ u_{j}}=\precsim u_{j}$, since $u_{j}(x) \leq u_{j}(y)$ if and only if $\phi_{j} \circ u_{j}(x) \leq \phi_{j} \circ u_{j}(y)$ for every $x, y \in X$ which means that $u_{j}$ and $\phi_{j} \circ u_{j}$ define the same total preorder in $\Re$. Therefore, $F\left(u_{i}\right)=F\left(\phi_{i} \circ u_{i}\right)$ and so $F$ satisfies information invariance with respect to cardinal measurability. It remains to prove that $F$ is continuous. To that end, observe that $\left\{\left(\left(a_{i}\right),\left(b_{i}\right)\right) \in \mathbb{R}^{n} \times \mathbb{R}^{n}\right.$ : there exist $\left(u_{i}\right) \in U^{n}, x, y \in X$ with $u_{i}(x)=a_{i}, u_{i}(y)=b_{i}$ and $\left.x F\left(u_{i}\right) y\right\}=\left\{\left(\left(a_{i}\right),\left(b_{i}\right)\right) \in\right.$ $\mathbb{R}^{n} \times \mathbb{R}^{n}$ : there exist $\left(u_{i}\right) \in U^{n}, x, y \in X$ with $u_{i}(x)=a_{i}, u_{i}(y)=b_{i}$ and $\left.u_{j}(x) \leq u_{j}(y)\right\}=\left\{\left(\left(a_{i}\right),\left(b_{i}\right)\right) \in \mathbb{R}^{n} \times \mathbb{R}^{n}: a_{j} \leq b_{j}\right\}$, which, clearly, is a Euclidean-closed subset of $\mathbb{R}^{2 n}=\mathbb{R}^{n} \times \mathbb{R}^{n}$.

To prove the converse, consider the binary relation $\precsim *$ defined on $\mathbb{R}^{n}$ as follows: Given $a=\left(a_{1}, \ldots, a_{n}\right)$ and $b=\left(b_{1}, \ldots, b_{n}\right) \in \mathbb{R}^{n}$, then $a \precsim^{*} b$ if and only if there exist $x, y \in X$ and a profile $\left(u_{i}\right) \in U^{n}$ such that for every $i \in N$ it holds that $u_{i}(x)=a_{i}, u_{i}(y)=b_{i}$ and $x F\left(u_{i}\right) y$. Since $F$ satisfies the binary independence of irrelevant alternatives condition, and $X$ contains at least three elements, it can be shown that $\precsim *$ is a total preorder that "generates" $F$. Indeed, by definition, $\precsim^{*}$ is obviously reflexive. It is total since the domain of $F$ is $U^{n}$. Let us see that it is transitive too. To that end, let $a=\left(a_{i}\right), b=$ $\left(b_{i}\right), c=\left(c_{i}\right) \in \mathbb{R}^{n}$ such that $a \precsim^{*} b$ and $b \precsim^{*} c$. We want to show that $a{ }^{*} c$. Since $a \precsim^{*} b$ there are $x, y \in X$ and a profile of utility functions $\left(u_{i}\right) \in U^{n}$ such that $u_{i}(x)=a_{i}, u_{i}(y)=b_{i}$ for all $i$, and $x F\left(u_{i}\right) y$. Similarly, since $b \precsim^{*} c$, there are $z, t \in X$ and a profile of utility functions $\left(v_{i}\right) \in U^{n}$ such that $v_{i}(z)=b_{i}$, $v_{i}(t)=c_{i}$ for all $i$ and $z F\left(v_{i}\right) t$. Consider a profile of utility functions $\left(w_{i}\right) \in U^{n}$ such that, for each $i \in N, w_{i}(x)=a_{i}, w_{i}(y)=w_{i}(z)=b_{i}$ and $w_{i}(t)=c_{i}$. Note that such a profile $\left(w_{i}\right) \in U^{n}$ does exit since $U$ consists of all functions from $X$ into $\mathbb{R}$ and, by hypothesis, $X$ has at least three elements (note that it might be that $y=z$ ). Then, by the binary independence of irrelevant alternatives condition and the fact that $x F\left(u_{i}\right) y$ it follows $x F\left(w_{i}\right) y$. Similarly, we have 
that $y F\left(w_{i}\right) t$. Now, $F\left(w_{i}\right) \in \Re$, hence it is a transitive binary relation defined on $X$. Thus, $x F\left(w_{i}\right) t$, for such a profile $\left(w_{i}\right) \in U^{n}$ for which $w_{i}(x)=a_{i}$ and $w_{i}(t)=c_{i}$. Therefore, by definition of $\precsim^{*}, a \precsim^{*} c$. So, $\precsim^{*}$ is transitive. In addition, it should be noted that, since $F$ is Paretian, $\precsim^{*}$ is non-trivial and increasing $^{5}$. Moreover, for every $a, b \in \mathbb{R}^{n}$ such that $a \ll b$ it holds that $a \prec^{*} b$.

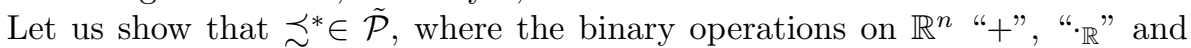
"*" are the usual ones defined componentwise.

Indeed, $\precsim^{*}$ is non-zero since $1_{n}$ is the identity of $\mathbb{R}^{n}$ with respect to "*" and $0_{n} \prec^{*} 1_{n}$, hence $\precsim^{*}$ is non-trivial, because $F$ is Paretian. Continuity of $\precsim^{*}$, referred to the Euclidean topology in $\mathbb{R}^{n}$, follows directly from the continuity of $F$ (see in Section 2 the equivalent formulation of continuity for a total preorder). Let us prove now that $\precsim^{*}$ is translation-invariant. To that end, let $a=\left(a_{i}\right), b=\left(b_{i}\right), c=\left(c_{i}\right) \in \mathbb{R}^{n}$ such that $a \precsim^{*} b$. Then there are $x, y \in X$ and a profile of utility functions $\left(u_{i}\right) \in U^{n}$ such that $u_{i}(x)=a_{i}, u_{i}(y)=b_{i}$ for all $i$ and $x F\left(u_{i}\right) y$. For every $i$, consider the functions $v_{i}(z)=u_{i}(z)+c_{i}$ for all $z \in X$. By information invariance with respect to cardinal measurability, it holds that $F\left(v_{i}\right)$ coincides with $F\left(u_{i}\right)$. In particular, $x F\left(v_{i}\right) y$ or, equivalently, $\left(v_{i}(x)\right) \precsim^{*}\left(v_{i}(y)\right)$. Thus, $a+c=\left(a_{i}+c_{i}\right) \precsim^{*} b+c=\left(b_{i}+c_{i}\right)$.

Similarly, let us prove that $\precsim^{*}$ is homothetic. To see this, let $a=\left(a_{i}\right), b=$ $\left(b_{i}\right) \in \mathbb{R}^{n}$, with $0 \leq \lambda$, such that $a{ }^{*} b$. If $\lambda=0$, obviously $\lambda \cdot a \precsim^{*} \lambda \cdot b$. So, suppose that $0<\lambda$. Then, there are $x, y \in X$ and a profile of utility functions $\left(u_{i}\right) \in U^{n}$, such that $u_{i}(x)=a_{i}, u_{i}(y)=b_{i}$ for all $i$ and $x F\left(u_{i}\right) y$. For each $i$, consider the (utility) function $v_{i}=\lambda u_{i}$. By information invariance with respect to cardinal measurability, it holds that $F\left(v_{i}\right)$ and $F\left(u_{i}\right)$ coincide. In particular, $x F\left(v_{i}\right) y$ or, equivalently, $\left(v_{i}(x)\right) \precsim *\left(v_{i}(y)\right)$. Thus, $\lambda \cdot a=\left(\lambda a_{i}\right) \precsim^{*} \lambda \cdot b=\left(\lambda b_{i}\right)$. Notice that, by translation invariance and homotheticity of $\precsim^{*}$ it follows, as in the proof of Theorem 5.12, that $I\left(0_{n}\right)=\left\{z \in \mathbb{R}^{n}: z \sim^{*} 0_{n}\right\}$ is a vector subspace of $\mathbb{R}^{n}$.

The fact that $\precsim^{*}$ is multiplicative-invariant, with respect to the binary operation $*$ defined componentwise (i.e., $\left(a_{i}\right) *\left(b_{i}\right)=\left(a_{i} b_{i}\right)$ for every $\left.\left(a_{i}\right),\left(b_{i}\right) \in \mathbb{R}^{n}\right)$, is a little more tricky. Since $\precsim *$ is translation-invariant it should be noted that, in order to prove that $\precsim^{*}$ is multiplicative-invariant, it is sufficient to show that for any pair $a, b \in \mathbb{R}^{n}$ such that $0_{n} \precsim * a, b$ it holds that $0_{n} \precsim^{*} a * b$. To show this it will be useful to observe the following facts:

(1) Given any $a=\left(a_{i}\right) \in \mathbb{R}^{n}$, such that $0_{n} \precsim^{*} a=\left(a_{i}\right)$, it holds that $0_{n} \precsim^{*} a * c$ for all $0_{n} \ll c=\left(c_{i}\right)$ (In particular, this will imply that if $0_{n} \sim^{*} a$, then $0_{n} \sim^{*} a * c$ for all $\left.0_{n} \ll c\right)$. Indeed, since $0_{n} \precsim * a$, there are $x, y \in X$ and a profile of utility functions $\left(u_{i}\right) \in U^{n}$, such that $u_{i}(x)=0$, $u_{i}(y)=a_{i}$ for all $i$ and $x F\left(u_{i}\right) y$. For each $i$, consider the (utility) function

\footnotetext{
${ }^{5}$ A total preorder $\precsim$ defined on $\mathbb{R}^{n}$ is said to be increasing if $x \leq y$ implies $x \precsim y$ for every $x, y \in \mathbb{R}^{n}$ (Here, $x=\left(x_{1}, \ldots, x_{n}\right) \leq y=\left(y_{1}, \ldots, y_{n}\right)$ means that $x_{i} \leq y_{i}$ for all $\left.i \in N\right)$. "«" will stand for the strict partial order in $\mathbb{R}^{n}$ (i.e., $x \ll y$ if and only if $x_{i}<y_{i}$ for all $i \in N$ ). The zero vector in $\mathbb{R}^{n}$ and the vector with all its coordinates equal one will be denoted by $0_{n}$ and $1_{n}$, respectively.
} 
$v_{i}=c_{i} u_{i}$. By information invariance with respect to cardinal measurability, it holds that $F\left(v_{i}\right)$ and $F\left(u_{i}\right)$ coincide. In particular, $x F\left(v_{i}\right) y$ or, equivalently, $\left(v_{i}(x)\right) \precsim^{*}\left(v_{i}(y)\right)$. Thus, $0_{n} \precsim^{*} a * c=\left(a_{i} c_{i}\right)$.

(2) Let then $a=\left(a_{i}\right) \in \mathbb{R}^{n}$ such that $0_{n} \precsim^{*} a$ and denote by $\left(e^{i}\right)_{i \in N}$ the canonical basis in $\mathbb{R}^{n}$. Let us see that, for each $i \in N, 0_{n} \precsim^{*} a_{i} \cdot e^{i}$ and, in addition, if $a_{i} \leq 0$, then $0_{n} \sim^{*} a_{i} \cdot e^{i}$. Indeed, let $i \in N$ be fixed. If $a_{i} \geq 0$, then the fact that $0_{n} \precsim^{*} a_{i} \cdot e^{i}$ follows directly since $\precsim^{*}$ is increasing and so $0_{n} \leq a_{i} \cdot e^{i}$ implies that $0_{n} \precsim * a_{i} \cdot e^{i}$. If $a_{i}<0$ the argument requires a further consideration. On the one hand, the fact that $a_{i} \cdot e^{i} \precsim^{*} 0_{n}$ follows by the previous reasoning again. To prove that $0_{n} \precsim^{*} a_{i} \cdot e^{i}$ consider, for every $m \in \mathbb{N}$, the vector $c^{m}=\left(c_{k}^{m}\right) \in \mathbb{R}^{n}$ defined as follows: $c_{k}^{m}=\frac{1}{m}$, if $k \neq i$ and $c_{i}^{m}=1$. Clearly, the sequence $\left(c^{m}\right)_{m=1}^{\infty}$ converges to $e^{i}$, as $m$ goes to $\infty$, and therefore the sequence $\left(a * c^{m}\right)_{m=1}^{\infty}$ converges to $a_{i} \cdot e^{i}$, as $m$ goes to $\infty$. Moreover, for every $m \in \mathbb{N}, 0_{n} \ll c^{m}$ and, since $0_{n} \precsim * a$, it follows also, from the argument of the previous paragraph, that $0_{n} \precsim * a * c^{m}$. Now, the continuity of $\precsim^{*}$ clearly implies that $0_{n} \precsim^{*} a_{i} \cdot e^{i}$. This fact, in combination with $a_{i} \cdot e^{i} \precsim^{*} 0_{n}$, yields $0_{n} \sim^{*} a_{i} \cdot e^{i}$. Thus, $0_{n} \sim^{*} a_{i} \cdot e^{i}$ provided that $a_{i} \leq 0$ (the case $a_{i}=0$ is trivial).

Let then $a=\left(a_{i}\right), b=\left(b_{i}\right) \in \mathbb{R}^{n}$ such that $0_{n} \precsim^{*} a, b$ and let us prove that $0_{n} \precsim^{*} a * b$. Notice that $a * b=\sum\left(a_{i} b_{i}\right) \cdot e^{i}$. Let us show that, for all $i \in N, 0_{n} \precsim^{*}\left(a_{i} b_{i}\right) \cdot e^{i}$. Then, the result would follow from the fact that $\precsim^{*}$ is translation-invariant. Let $i \in N$ be fixed. If $0 \leq a_{i} b_{i}$, then $0_{n} \precsim *\left(a_{i} b_{i}\right) \cdot e^{i}$ since $\precsim^{*}$ is increasing. It remains to analyze what occurs if $a_{i} b_{i}<0$. Assume that $a_{i}<0$ and $b_{i}>0$, the other case (i.e., $a_{i}>0$ and $b_{i}<0$ ) being similar. Then, by the argument above, $0_{n} \sim^{*} a_{i} \cdot e^{i}$, i.e., $a_{i} \cdot e^{i} \in I\left(0_{n}\right)$. Now, since $I\left(0_{n}\right)$ is a vector subspace of $\mathbb{R}^{n}$, it follows that $\left(a_{i} b_{i}\right) \cdot e^{i}=b_{i} \cdot\left(a_{i} \cdot e^{i}\right) \in I\left(0_{n}\right)$. In particular, we have that $0_{n} \precsim^{*}\left(a_{i} b_{i}\right) \cdot e^{i}$ as desired.

Thus, ${ }^{*}$ is multiplicative-invariant. Hence all the conditions of Remark 5.15 are satisfied and therefore $\precsim^{*}$ is a projective total preorder on $\mathbb{R}^{n}$. This clearly implies that $F$ is a strongly dictatorial social welfare functional.

Remark 6.2. In the proof of Theorem 6.1 above we have used the fact that, with respect to the usual binary operations " $+"$, " $\mathbb{R}$ " and " $*$ ", defined componentwise, $I\left(0_{n}\right)$ is a vector subspace of $\mathbb{R}^{n}$. In fact, as it was proved in the proof of Theorem 5.12, $I\left(0_{n}\right)$ is also an ideal of $\mathbb{R}^{n}$. So, a fortiori, $I\left(0_{n}\right)$ is of the form $I\left(0_{n}\right)=\sum_{i \in N, i \neq j} \lambda_{i} e^{i}$ for some $j \in N$. But this fact is a consequence of Theorem 5.12 and Remark 5.15

As a direct consequence of the previous Theorem 6.1, we obtain the following impossibility result.

Corollary 6.3. Suppose that $X$ contains at least three elements. Then there is no continuous social welfare functional $F: U^{n} \rightarrow \Re$ that is Paretian, satisfies the binary independence of irrelevant alternatives condition, information invariance with respect to cardinal measurability, and anonymity. 
Acknowledgements. Thanks are given to an anonymous referee for the excellent revision done. His/her suggestions and comments have led to substantial improvements in the manuscript.

This work has been supported by the research projects MTM 2007-62499, ECO2008-01297 and MTM2009-12872-C02-02 (Spain).

\section{References}

[1] C. D. Aliprantis and K. C. Border, Infinite Dimensional Analysis, A Hitchhiker's guide, Springer, Berlin, 1999.

[2] G. Birkhoff, A note on topological groups, Compos. Math. 3 (1936), 427-430.

[3] D. S. Bridges and G. B. Mehta, Representation of Preference Orderings, Springer, Berlin, 1995.

[4] M. J. Campión, J. C. Candeal, and E. Induráin, The existence of utility functions for weakly continuous preferences on a Banach space, Math. Social Sci. 51 (2006), no. 2, $227-237$.

[5] _ On Yi's extension property for totally preordered topological spaces, J. Korean Math. Soc. 43 (2006), no. 1, 159-181.

[6] _ Preorderable topologies and order-representability of topological spaces, Topology Appl. 156 (2009), no. 18, 2971-2978.

[7] _ Semicontinuous planar total preorders on non-separable metric spaces, J. Korean Math. Soc. 46 (2009), no. 4, 701-711.

[8] M. J. Campión, J. C. Candeal, E. Induráin, and G. B. Mehta, Representable topologies and locally connected spaces, Topology Appl. 154 (2007), no. 10, 2040-2049.

[9] J. C. Candeal, C. Hervés, and E. Induráin, Some results on representation and extension of preferences, J. Math. Econom. 29 (1998), no. 1, 75-81.

[10] J. C. Candeal and E. Induráin, Utility functions on chains, J. Math. Econom. 22 (1993), no. $2,161-168$

[11] _ A note on linear utility, Econom. Theory 6 (1995), no. 3, 519-522.

[12] _ Lexicographic behaviour of chains, Arch. Math. (Basel) 72 (1999), no. 2, 145152.

[13] J. C. Candeal, E. Induráin, and G. B. Mehta, Order preserving functions on ordered topological vector spaces, Bull. Austral. Math. Soc. 60 (1999), no. 1, 55-65.

[14] Utility functions on locally connected spaces, J. Math. Econom. 40 (2004), no. 6, 701-711.

[15] J. C. Candeal, E. Induráin, and J. A. Molina, Numerical representability of ordered topological spaces with compatible algebraic structure, To appear in Order. DOI 10.1007/s 11083-011-9202-8.

[16] H. H. Corson, The weak topology of a Banach space, Trans. Amer. Math. Soc. 101 (1961), 1-15

[17] G. Debreu, Representation of a preference ordering by a numerical function, In R. Thrall, C. Coombs and R. Davies (Eds.), Decision processes, John Wiley. New York, 1954.

[18] J. Dugundji, Topology, Allyn and Bacon, Boston, 1966.

[19] S. Eilenberg, Ordered topological spaces, Amer. J. Math. 63 (1941), 39-45.

[20] R. Engelking, General Topology, Revised and Completed Edition. Heldermann Verlag. Berlin, 1989.

[21] M. Estévez and C. Hervés, On the existence of continuous preference orderings without utility representations, J. Math. Econom. 24 (1995), no. 4, 305-309. 
[22] M. Fleurbaey and P. J. Hammond, Interpersonally comparable utility, Chapter 21 of the Handbook of Utility Theory (Vol. 2), Edited by Barberà S, Hammond PJ and Seidl C, Kluwer Academic Publishers. Amsterdam, 2004.

[23] L. Fuchs, Partially Ordered Algebraic Systems, Pergamon Press, Reading, Massachusetts, 1963.

[24] V. Gutev, Weak orderability of second countable spaces, Fund. Math. 196 (2007), no. $3,275-287$.

[25] G. Herden, Topological spaces for which every continuous total preorder can be represented by a continuous utility function, Math. Social Sci. 22 (1991), no. 2, 123-136.

[26] G. Herden and A. Pallack, Useful topologies and separable systems, Appl. Gen. Topol. 1 (2000), no. 1, 61-82.

[27] S. Kakutani, Über die Metrisation der topologischen Gruppen, Proc. Imp. Acad. 12 (1936), no. 4, 82-84.

[28] J. L. Kelley, General Topology, Van Nostrand. New York, 1955.

[29] D. J. Lutzer and H. R. Bennet, Separability, the countable chain condition and the Lindelöf property on linearly ordered spaces, Proc. Amer. Math. Soc. 23 (1969), 664667.

[30] J. R. Munkres, Topology, (2nd ed.), Prentice Hall. New York, 2000.

[31] S. Purisch, A history of results on orderability and suborderability, In Handbook of the history of General Topology, Vol. 2, pp. 689-702. Kluwer. Dordrecht, 1998.

[32] C. E. Rickart, General Theory of Banach Algebras, Van Nostrand. Princeton, N.J., 1960.

[33] H. H. Schaefer, Topological Vector Spaces, (3rd ed.), Springer Verlag. New York, 1971.

[34] P. Simon, A connected, not separably connected metric space, Rend. Istit. Mat. Univ. Trieste 32 (2001), suppl. 2, 127-133.

[35] L. A. Steen and J. A. Jr. Seebach, Counterexamples in Topology, (2nd ed.), Springer Verlag. New York, 1978.

[36] J. van Dalen and E. Wattel, A topological characterization of ordered spaces, General Topology and Appl. 3 (1973), 347-354.

[37] G. Yi, Continuous extension of preferences, J. Math. Econom. 22 (1993), no. 6, 547-555.

MARÍA Jesús CAMPióN

Departamento de Matemáticas

Universidad Pública de Navarra

E-31006 PAmplona, Spain

E-mail address: mjesus.campion@unavarra.es

Juan Carlos Candeal

Departamento de Análisis ECONÓmico

UNIVERSIDAD DE ZARAGOZA

E-50005 ZARAGOZA, SPAIN

E-mail address: candeal@unizar.es

ESTEBAN INDURÁIN

DePARTAMENTo DE MATEMÁticas

UNiversidad PÚBlicA DE NAVARRA

E-31006 PAmplona, Spain

E-mail address: steiner@unavarra.es

Ghanshyam Bhagvandas Mehta

DEPARTMENT OF ECONOMICS

UNIVERSITY OF QUEENSLAND

Brisbane 4072, Australia

E-mail address: mehtagb@gmail.com 